\title{
$\rightarrow$ \\ Hepatocellular carcinoma and liver transplantation
}

Liver transplantation has consolidated itself as a highly effective treatment

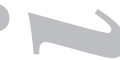
modality for patients with end-stage chronic liver disease, fulminant liver failure, and hepatocellular carcinoma (HCC), with overall survival rates at one year around $80 \%$, and $95 \%$ in a number of groups (chronic cholestatic liver disease, patients with small-size hepatocellular carcinoma). In this respect the success of the Spanish model, with its first transplant performed in 1984 by Dr. Margarit (in memoriam) and Dr. Jaurrieta, is now greater than that of European countries that initiated their transplantation-related activities 5, 9, and 16 years earlier (Italy, France, and the United Kingdom, respectively). In 2004 Spain was first among European countries in absolute number of transplants (11,660, followed by France with 10,916 and the UK with 10,252, according to data from the European Transplant Registry).

$\mathrm{HCC}$ is the most common primary malignancy, and represents around $12 \%$ of transplant indications (data from the same Registry). Spain has evolved from 7\% in 1987-1989 to $24 \%$ in 2002-2004, reaching $34.4 \%$ among recipients older than 60 years of age (data from the ONT, 2005 report); hence it represents a serious problem that we shall still confront in the future.

Most patients are infected with $\mathrm{HCV}$ or $\mathrm{HBV}$, and suffer from hemochromatosis or ethylic cirrhosis (1). The presence of replicative HBV infection, and particularly the association of HCV with HBV markers (positive anti-HBc and anti-HBs antibodies), which is seen in more than $55 \%$ of patients with HCC versus $12 \%$ of control subjects, notably increases the risk for HCC development.

Incidence ranges from 5-15 cases per 100,000 population in Europe and the USA to 27-36 per 100,000 in East Asia. Liver cirrhosis is identified in $90 \%$ of cases at diagnosis, with a prevalence of 5\% in compensated patients and of $15-20 \%$ in decompensated patients. Its annual incidence is $3-6 \%$, and is controversial whether previous treatment with IFN may reduce the risk of HCC. A transplant is doubly curative in these patients, as both the HCC and cirrhosis are removed with this procedure. It is for this reason that the treatment of choice for patients with a stage-I or -II HCC should be a transplant in the absence of contraindications or older age. The discouraging results obtained during the early 1990s, with a 5-year survival of $18 \%$, led to a better selection of patients, with efforts being focused on early diagnosis, appropriate staging, and combined transplant and adjuvant chemotherapy, or neoadjuvant chemotherapy before transplantation. Survival, as based on 6,547 patients in the European Registry through December 2004, is currently 78, 54, and 42\% at 1, 5, and 10 years respectively, with survival rates seen from year 3 on being lower versus the remaining etiologies ( $76 \%$ for cirrhosis, $63 \%$ for fulminant liver failure, and $62 \%$ for HCC). A number of causes might be related to mortality at 3 and 12 months, particularly including fulminant liver failure as a cause of transplant, donor 
age older than 60 years, split or reduced graft, total ischemia time longer than 13 hours, and low UNOS score. However, alcoholic cirrhosis, HCC, hepatitis C, hepatitis $\mathrm{B}, \mathrm{PBC}$, donor older than 40 , and experience (more than 70 transplants per year) are associated with lower mortality (2).

Accepted liver transplant indications for patients with HCC include one nodule $<5 \mathrm{~cm}$ or up to 3 nodules $<3 \mathrm{~cm}$ (so-called Milan criteria) $(3,4)$. UCSF has recently suggested extended criteria, namely one nodule $<6,5 \mathrm{~cm}$ or multiple nodules $<4,5 \mathrm{~cm}$ each, or $>8 \mathrm{~cm}$ together (5). A sole exception would be fibrolamellar carcinoma, whose slow progression would allow a transplant with greater lesions. Contraindications are associated with widespread tumor disease, distant metastasis, or vascular invasion. Overall, except for cirrhotic patients in a Child-A stage with normal bilirubin, more than 100,000 platelets, and no esophageal varices, which may be considered eligible for surgery, all patients with this condition and younger than 65 years of age should be considered potential candidates for LT.

To improve post-transplant survival the first problem to be solved is an adequate selection of patients in an attempt to assimilate long-term survival rates in patients with $\mathrm{HCC}$ to those seen in other etiologies, as post-transplant $\mathrm{HCC}$ recurrence rates are in fact $10-15 \%$ (6), and $46 \%$ of patients transplanted for $\mathrm{HCC}$ who die in our country do so from a relapsing tumor (ONT data, 2005). To answer this question it is essential that the accuracy extent of current imaging techniques for post-transplant tumor staging be known, as well as which classification system is more reliable when it comes to decide a patient's therapy. Unfortunately, the sensitivity and specificity of the three most commonly used imaging techniques (ultrasounds, CT, and contrast MRI) are rather low. Maybe ultrasonography is more sensitive for the diagnosis of small-size tumors, whereas MRI is more specific for lesions greater than $2 \mathrm{~cm}$. A number of studies find patient understaging rates to be as high as $20 \%$, some of them with pre-transplant diagnostic accuracy percentages as low as $14 \%$, and a sensitivity of 0 and $21 \%$ for 1 - and 2-cm tumors, respectively (7). Regarding the tumor classification system a higher level of consensus does seem to exist, and the model suggested by Hospital Clínico de Barcelona seems to be the best for patient staging regarding subsequent treatment from a prognostic standpoint. Such model has been accepted by the American Association for the Study of Liver Diseases $(4,8)$, and prospectively validated in Italy $(9)$.

A second issue to consider when trying to modify post-transplant recurrence rates is whether patients on the waiting list should be treated using a standard prioritization system, or a system prioritizing patients with tumors greater than $2 \mathrm{~cm}$, as is the case with the MELD system. There is no clear evidence that any treatment should be used for patients with a probability for transplant shorter than 6 months (10); hence a most adequate option -if possible- would be to increase organ availability in order to reduce waiting lapses, a theoretical rather than practical issue except for living donor liver transplantation, or to prioritize these patients with an organ distribution regimen allowing them to receive a transplant before being excluded from the waiting list because of disease progression (11). From 1998 on, and with subsequent amendments, these patients have started to be prioritized in the USA, assigning them a MELD score of 22 (T2, Milan criteria) to avoid the risk for widespread disease at LT, this score being increased every 3 months while patients remain on the waiting list. Some teams have started to follow this guideline in Spain, but using slightly lower scores. Tumor molecular characteristics may provide information not only from a prognostic perspective, but also for therapy target development (12). 
A third option to reduce post-transplant tumor recurrence relates to the immunosuppressive treatment these patients should receive, and to whether they may benefit from some relapse-preventing strategy. Excessive immunosuppression seems to favor tumor relapse (6), whereas some sirolimus-, and possibly everolimus-based therapy regimens may reduce or even delete tumor recurrence (13). Further well-designed, prospective studies are needed allowing to demonstrate the benefits provided by these immunosuppression regimens in patients with a high risk for relapse.

The article by Pérez-Luque et al. (14) in this same issue of the Spanish Journal of Gastroenterology retrospectively assesses survival in their cohort of patients transplanted for this condition. Patients were selected according to internationally accepted criteria for their inclusion on the waiting list, and no patients were accepted that might comply with the so-called expanded criteria. The extension study consisted of preoperative Doppler ultrasounds, chest CT, and bone scintigraphy. MRI currently plays a preponderant role in this study because of a higher sensitivity for nodules greater than $2 \mathrm{~cm}$, as previously reported. Results highlight a high incidence of incidental $\mathrm{HCC}(32.1 \%)$, probably related to the low sensitivity of imaging techniques as a whole, which needs to be improved despite the relevant advances in this field so far. Indeed, while non-invasive diagnostic criteria- based on the presence of a nodule greater than $2 \mathrm{~cm}$ in a CT or MRI scan consistent with HCC (hypervascular lesion during the arterial phase with portal washout), and compatible CT plus MRI scans for nodules $1-2 \mathrm{~cm}$ in size- seem to predominate of late (4), patients are commonly found in daily practice where -despite a high clinical or ultrasonographic suspicion- the presence of an HCC cannot be demonstrated by CT or MRI until a few months later, in addition to an obvious lack of correlation between pretransplant diagnosis and pathology. Such uncertainty prompts us to greater caution in all respects, both when indicating and not indicating a liver transplant. This is why cytology plays a very important role in diagnosing this type of patients, despite a theoretical dissemination risk (15) that has nor been clearly elucidated yet (16).

As with most transplant groups, the most common etiology was HCV -more than $50 \%$ of patients, with a low mean age at 53 years, and a gender distribution of 9:1 (males:females). In all, 34.6\% died, with the most common mortality cause being tumor relapse with a recurrence rate of $12.3 \%$, similar to other series (17); HCV relapse was the second most common cause of death. Survival at 1,5 , and 10 years was 80,61 , and 52\%, respectively, similar to cases in the European Registry. However, the poorer outcome for incidental HCC is notable, as this tumor is usually small, and this criterion -tumor size- is considered a positive prognostic factor (18), with patients in this subgroup having survivals superimposable to those of patients receiving a transplant for a non-tumor disease (4). No post-transplant follow-up scheme has been found with a lower recurrence rate. Patients with HCC and elevated alpha-fetoprotein may be monitored by periodically measuring the latter parameter; but such monitoring has not been seen to allow an earlier diagnosis, and does not seem to modify a relapsing patient's natural history. Relapse usually develops within 2 years post-transplant (6), with a mean survival of few months following diagnosis. In our experience with 547 transplant patients, 83 for liver carcinoma, recurrence rates in patients with expalnted livers that met Milan criteria were $10 \%$, while $20 \%$ of understaged patients had a recurrence rate of $15 \%$, with recurrence developing within one year post-transplant in $80 \%$ of cases, with a mean survival of only 2 months afer diagnosis. Survival in the series by Pérez-Luque et al. is surprising, with 5 years for patients with established $\mathrm{HCC}$, and a mean of 7 years for pa- 
tients with incidental HCC, even if most relapsing patients died within 3 years posttransplant, which is more consistent with data reported thus far. Various criteria for poor prognosis have been suggested, including AFP elevation, tumor size, differentiation extent, etc. In the absence of extrahepatic dissemination, those that are most consistent and are used by most reports include vascular macro- and micro-invasion, and presence of peritumoral satellite nodes, both directly related to tumor size. Tumor differentiation has been related to vascular invasion inducibility (19). The finding that patients with a short survival time have an increased expression of antiapoptosis genes, histone modification genes, or genes associated with increased cell proliferation may identify biologically distinct HCC subclasses, which would help in a better selection of patients, and in the search for pre- or post-transplant strategies to effectively reduce tumor recurrence, and hence improve survival in our patients (20).

M. García and R. Bárcena

Unit of Liver Transplantation. Service of Gastroenterology. "Ramón y Cajal” Hospital. Madrid, Spain 